\title{
Knowledge and Practices among Female Nurses on Insertion and Care of Indwelling Urinary Catheter to Female Patients in a Selected Teaching Hospital in Sri Lanka
}

\author{
Priyantha Rohan ${ }^{1}$ and Thamara D. Amarasekara2* \\ ${ }_{1,2}$ Nursing and Midwifery, Faculty of Allied Health Sciences, University of \\ Sri Jayewardenepura, Sri Lanka
}

\section{Abstract}

Globally, catheter-associated urinary tract infection (CAUTI) is one of the most common hospital-acquired infections. By following proper guidelines and recommended practice regarding insertion and care of indwelling urinary catheter, one could prevent and reduce CAUTI. Knowledge and practices among nurses regarding the insertion and care of indwelling urinary catheter in female patients are highly essential to minimize complications related to indwelling urinary catheter among female patients. There are limited data available on this phenomenon in Sri Lanka. The purpose of this study was to determine knowledge and practices among nurses regarding the insertion and care of indwelling urinary catheter in female patients at a selected teaching hospital. A descriptive cross-sectional design was conducted among conveniently selected 122 nurses from a teaching hospital. A pre-tested $(\mathrm{n}=10)$, a semi-structured questionnaire, was used to collect data. Ethical approval was obtained from the ethics review committees of the Faculty of Medical Sciences, University of Sri Jayewardenepura, and Colombo South Teaching Hospital, Sri Lanka. Findings revealed that only $36 \%$ of the participants had a satisfactory

*Corresponding should be addressed to Dr. T. D. Amaraekara, Faculty of Allied Health Sciences, University of Sri Jayewardenepura, Sri Lanka

Email: thamara@sjp.ac.lk

(D) https://orcid.org/0000-0003-0191-0973

(Received 24th October 2018; Revised 13 th March 2019; Accepted 19th June 2019) (C) OUSL 
level of knowledge on indwelling urinary catheter insertion. Most of the participants $(79.5 \%)$ had a satisfactory level of practices regarding indwelling urinary catheter insertion. Majority of the participants $(82 \%)$ had an unsatisfactory level of knowledge regarding indwelling urinary catheter care. Most of the participants $(78.7 \%)$ had a satisfactory level of practices regarding indwelling urinary catheter care. In conclusion, the majority of the participants had an unsatisfactory level of knowledge regarding indwelling urinary catheter insertion and care. There is a need for education on indwelling urinary catheterization and care for nurses in Sri Lanka.

Keywords: Nurses, Knowledge, Practices, Catheter care, Sri Lanka

\section{Introduction}

A urinary catheter is an important medical device. The purposes of urinary catheters are many. For example, to relieve urinary retention, to monitor accurate urinary output, to instill medications, to manage and maintain urinary system during surgical procedure, to establish bladder irrigation for management of haematuria, to manage fistula and promote healing, to conduct investigative procedures, to preserve skin integrity, to monitor urine output, to relieve urinary retention and diagnosing pathology in the lower urinary tract (Urology Nursing Working Group, 2013). Urinary catheterization is used after some of the urological surgery such as distal to mid-shaft hypospadias to enable measurement of intra- and postoperative urine output and to prevent possible acute urinary retention after surgery due to swelling and pain (Hosseinpour et al., 2014).

A hospital-acquired infection (HAIs) causes serious outcomes to a patient, family and the health care system, among them, are: long duration of hospital stay, long-term disability, developing of resistance to anti-micro bacterial drugs and preventable deaths and financial inconvenience to the healthcare system (World Health Organization (WHO), 2010). The most affected site of HAIs is the urinary tract. Urinary tract infection (UTI) is the most common in HAI (Schumm and Lam, 2009). Indwelling urinary catheter (IUC) insertion is the primary cause of UTI, and this catheterization procedure gives one of the risk factors for UTI (Wickramasinghe and Dharmarathne, 2013). Urine culture is the most accurate measurement of patients having UTI or 
not (Smelter, 2005). The rate of developing bacteriuria for a patient with IUC is $3 \%-6 \%$ per day and there is a $50 \%$ chance of patients developing bacteriuria with IUC between 7 - 10 days (Pratt and Pellowe, 2010). If a patient's urine culture shows bacteriuria $>10^{5}$ colony forming units $(\mathrm{CFU}) / \mathrm{ml}$, it is considered a positive urine culture for non-catheterized patients. If bacteriuria shows $>10^{3}$ $\mathrm{CFU} / \mathrm{ml}$, it is considered as a positive urine culture for a catheterized patient (Schumm and Lam, 2009). Escherichia coli, Pseudomonas, Candida spp, Klebsiella and Enterococci are major pathogens for CAUTI. After catheterization, those pathogens enter the urinary tract by the extraluminal route or by the intraluminal route (Shimna et al., 2014). Proper knowledge and practices during the insertion and care for IUC will lead to reducing CAUTI and related complications. Inserting the IUC procedure is performed under aseptic technique by qualified nursing staff (Urology Nursing Working Group, 2013). When invasive urinary catheterization is not appropriately maintained in hospitalized patients, serious adverse effects can occur, including CAUTI (Bernard et al., 2012). Other than the CAUTI, long-term use of an indwelling urethral catheter can be associated with many complications like bladder spasms, hematuria, urethral erosion, urethritis, periurethral abscess, unprescribed removal, pain, fistula formation, stones, obstruction secondary to encrustation, and leakage (De Silva, 2011). CAUTIs and catheter-associated other complications can be prevented by following catheter care protocols developed from evidence-based practices (Newman, 2010).

The management of IUC including catheter insertion, removal, specimen collection and catheter care, is being mostly done by nurses. Nurses are the most responsible health care persons for urinary catheter management. Nurses are the persons who first notice about catheter-associated urinary tract infection among catheterized patients (Yoon et al., 2013). Majority of the female patients are urinarily catheterized by female nurses. However, IUC care for male and female patients is conducted by female nurses (Crown and Champman, 2000 cited in Govindamma, 2005).

Several preventive measures have been or observed in those requiring an indwelling catheterization. The most important infection control measure is the introduction of the closed catheter drainage system. 
In order to provide aseptic techniques during insertion and maintenance of the urinary catheter and drainage bag, health care workers wear gloves when manipulating or emptying the drainage bag and wash hands vigorously between patient contacts (Saint and Lipsky, 1999). There are several guidelines for urinary catheterization developed based on evidence. For example, CDC guidelines of the Infection Diseases Society of America (IDSA) describe various interventions for preventing CAUTI. Prevention of CAUTI has being identified through guideline-based knowledge. Healthcare workers must follow this when delivering care to the patients for prevention of CAUTI (Parida and Mishra, 2013). A nurse is the most responsible person for the prevention of CAUTI provided she follows knowledgebased practice regarding urinary catheterization and caring.

CDC documents presented more than 60 specific recommendations to prevent CAUTI. Most recommendations are for the prevention of CAUTI techniques specific to nursing practice such as the indication for insertion of catheters, a technique of insertion, management of obstruction, specimen collection, and documentation.

A study conducted by De Silva (2011) in Sri Lanka stated that urethral injuries have occurred due to several causes such as the use of the inappropriate size of the catheter, inappropriate technique of securing the catheter and inflation of the catheter insertion by untrained healthcare person. Ministry of Health statistics states that government expenditure for health was Rs 43,022 Million in 2007 and 9 per 100,000 population death occurred due to diseases of the urinary system in 2007 (Ministry of Health [MOH], 2008). Therefore, adequate knowledge and practices for insertion of urinary catheter and catheter care are paramount to reduce such consequences.

However, there is limited data available on nurses' knowledge and practices regarding the insertion and care of indwelling urinary catheter of female patients in Sri Lanka. Hence the present study is an effort to determine the knowledge and practices of nurses regarding the insertion and care of IUC. When nurses have sufficient knowledge of the insertion and care of IUC, catheter-associated complications can be reduced. This study hopes to encourage nurses to improve their knowledge and practices regarding the insertion and care of indwelling urinary catheter for female patients in Sri Lanka. 


\section{Justification}

IUC is an important invasive procedure for acute and chronic management of a patient with urinary problems. All the indwelling urinary catheter managements include catheter care, specimen collection and catheter removal (Yoon, et al., 2013). CAUTI is the most common hospital-acquired infection (HAI) (Fink, et al., 2012). An indwelling urinary catheter is the major associated cause for UTI (Schumm and Lam, 2009).

On the other hand, CAUTI is a financial burden on the patient and the healthcare system. CAUTI cause to increase terms of treatment and length of hospital stay. Globally there have been many types of research done to find strategies to prevent CAUTI and to produce guidelines for all kind of catheter management including cleaning, indwelling urinary catheterization, catheter care, catheter removal and specimen collection from the urinary catheter (Lo et al., 2014).

CDC and IDSA guidelines make recommendations to prevent CAUTI. Most of the catheter-related problems reported could be prevented or minimized with more attention to catheter management, early identification of problems and more evidence-based catheter practices (Wilde et al., 2012). Nurses are the most responsible persons for urinary catheter management. Nurses are the persons who notice CAUTI among catheterized patients (Yoon et al., 2013). Therefore, nurses' knowledge and practices regarding the insertion and care of indwelling urinary catheter are essential to reduce and prevent CAUTI. It could also reduce the duration of patients' hospital stay.

In Sri Lanka, there are more female nurses than male nurses $(\mathrm{MOH}$, 2012). Annually government enrols only $5 \%$ quota for male student nurses who register with government nursing training schools. Therefore, the majority of female nurses work in male wards. Generally, in male wards, medical officers perform the urinary catheterization procedure. Female nurses do not catheterize male patients. This is because of the inadequate distribution of male nurse in male wards and also due to Sri Lanka's gender-based cultural notions. Hence in female wards, female nurses are actively doing the insertion of urinary catheters and catheter care for female patients. 
Therefore, it is essential to identify whether the female nurses have adequate knowledge and practices regarding the insertion and care of indwelling urinary catheter of female patients. The findings of this study may be useful to improve nurses' knowledge and practices regarding indwelling urinary catheterization and catheter care. Furthermore, the findings will be useful to prevent or reduce hospitalacquired CAUTIs among Sri Lankan patients. Also, findings of the study may help to encourage further studies to prevent CAUTIs and improvement of guidelines for IUC insertion and infection control.

\section{Aim and design}

\section{Aim}

To determine the knowledge and practices of female nurses regarding the insertion and care of indwelling urinary catheter of female patients at a selected Teaching Hospital in Sri Lanka.

\section{Design, Setting \& Sample}

A descriptive cross-sectional design was used. This study was conducted at the Colombo South Teaching Hospital. Purposively selected $(n=122)$ female nurses from wards/units participated in this study. Inclusion criteria for the participants were: female nurses who worked in female surgical, medical, gynaecological, orthopaedic wards, surgical and medical ICUs at CSTH hospital with more than three months of working experiences and who would like to participate in this study. Working experience of more than three months was needed because to get experiences on IUC insertion and catheter care. Nurses who had less than three months of experiences were excluded from the study.

\section{Data collection \& data analysis}

Ethical approval was obtained from the ethics committees of the Faculty of Medical Sciences, University of Sri Jayewardenepura, $\mathrm{CSTH}$, and relevant authorities. All participants were informed verbally, and in writing about the study purpose and voluntary participation was encouraged. Written informed consent was obtained from all participants, and their privacy, confidentiality and rights were protected throughout.

A self-administered questionnaire was developed by the researchers 
based on extensive and intensive literature review. The questionnaire includes 03 sections: Section "A" included Demographics data (age, present working Ward, years of working experience, highest educational level), Section "B" included knowledge of nurses regarding insertion and care of indwelling urinary catheter of female patients and Section "C" included practices of nurses regarding insertion and care of indwelling urinary catheter of female patients. The questionnaire was pre-tested for ten staff nurses in other words, and relevant modifications were done accordingly. The questionnaire took less than 15 - 20 minutes to complete. The data were analyzed in a statistical package of social sciences (SPSS) 20th version for descriptive statistics such as frequency distribution, percentage, mean score and standard deviation.

Knowledge of nurses, regarding insertion of an indwelling urinary catheter of female patients, were assessed and for a correct answer on knowledge of insertion, 01 mark was given, for bivariate questions regarding knowledge of catheter care 01 mark was given for the correct answer and 0 for the incorrect answer. Then marks converted to a percentage and categorized into 03 groups: $0 \%-59 \%=$ Poor knowledge, $60 \%-72 \%=$ Fair knowledge, $73 \%-100 \%=$ Good knowledge. Knowledge of urinary catheter care questions was given a total of 2 marks and was categorized as follows:0 - 1 marks = Unsatisfactory and total 2 marks = Satisfactory.

Practices of nurses, regarding insertion and care of indwelling urinary catheter of female patients, were assessed as follows:(a) For a correct answer on practices of insertion 01 mark was given for each correct answer, (b) For bivariate questions 01 mark was given for the correct answer and 0 for an incorrect answer regarding practices of insertion. The results were categorized into 02 groups as $0-8$ Marks = Unsatisfactory, 8 - 12 Marks = Satisfactory. Practices on care were given a total of 3 marks, and then it was categorized as follows: 0-2 marks $=$ Unsatisfactory, a total of 3 marks $=$ Satisfactory

\section{Findings}

\section{Demographic characteristics of the participants}

According to Table-1, most of the participants (60.7\%) were less than or equal to 30 years of age $($ Mean=30.3). Only four participants $(3.3 \%)$ 
were graduates, and more than half of the participants (55.7\%) have 3 months -5 years of work experience.

Table 1. Demographic characteristics of the participants $(n=122)$

\begin{tabular}{lrr}
\hline Characteristics & Frequency & Percentage (\%) \\
\hline Age (years) & 74 & \\
Less than or equal to 30 & 40 & 60.7 \\
More than 30 & 8 & 32.8 \\
Not mentioned & & 6.6 \\
Mean=30.3 & & \\
SD=5.0 & & \\
Highest educational & & \\
qualification & 101 & 82.8 \\
Diploma & 13 & 10.7 \\
Undergraduate & 4 & 3.3 \\
Graduate & 4 & 3.3 \\
Not mentioned & & \\
& & 55.7 \\
Work experience (years) & 68 & 29.5 \\
3 months - 5 years & 36 & 12.3 \\
6 years - 10 years & 15 & 2.5 \\
> 10 years & 3 & \\
Not mentioned & & 27.0 \\
Working unit & & 22.1 \\
Surgical ward & 33 & 25.4 \\
Medical ward & 27 & 18.0 \\
ICU & 31 & \\
Gynaecological ward & 22 & \\
Orthopaedic ward & & \\
& & \\
\hline
\end{tabular}

\section{Knowledge and practices regarding IUC insertion}

Most of the participants could not correctly identify the correct answer for some questions related to knowledge on IUC insertion such as: what is catheterization procedure what is the average retention period of Foley catheter? The normal length of the female urethra is? Majority 
of the participants (Mean=5.1, SD=0.87) identified the answer for indications for catheterization. Most of the participants (Mean=5.7, $\mathrm{SD}=1.0)$ identified correct answers for documentation after the catheterization

Most of the participants had (more than 50\%) knowledge regarding the retained period of silicone catheter and catheter material. Majority of the participants (Mean=103.8) correctly answered the question of indications for catheterization. Less than half of the participants $(42.6 \%)$ have followed the aseptic technique before the catheterization procedure as a correct method. Nearly half of the participants (49.2\%) answered in the positive for following handwashing technique before the catheterization procedure. Most of the participants (Mean=86.5) had good practices regarding documentation after the IUC insertion. Participants' knowledge level on indwelling urinary catheter insertion was mostly (36.1\%) in the Fair Category (Table 2). Most of the participants $(79.5 \%)$ had a Satisfactory Level of practices regarding indwelling catheter insertion (Table 3).

Table 2. Overall knowledge of IUC insertion $(n=122)$

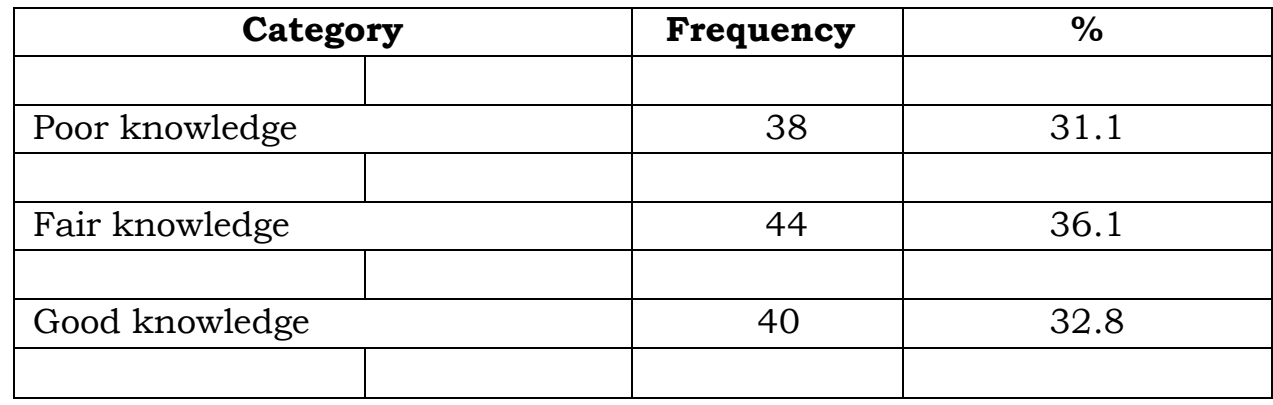


Table 3. - the Overall level of practices regarding IUC insertion $(n=122)$

\begin{tabular}{lcc}
\hline Category & Frequency & $\%$ \\
Satisfactory & 97 & 79.5 \\
Unsatisfactory & 25 & 20.5 \\
\hline
\end{tabular}

Most of the participants (63\%) who work in the medical wards had a good level of knowledge regarding the insertion of the indwelling urinary catheter. In gynaecological wards, only one subject had good knowledge level regarding IUC insertion. In orthopaedic wards, there was not any subject who possessed good knowledge regarding the insertion of the indwelling urinary catheter. In terms of practices of indwelling urinary catheter insertion, there was a big difference between a Satisfactory Level (7.4\%) and Unsatisfactory (92.6\%) within medical wards.

In relation to work experience, there was a good level of knowledge regarding IUC insertion among participants (52.8\%) who had 6-10 years of work experience. Most of the participants who had 3 months -5 years of work experience had fair knowledge (41.2\%) regarding IUC insertion. All most all participants $(100 \%)$ who were graduates had a good knowledge regarding indwelling urinary catheter insertion.

\section{Knowledge and practices regarding IUC care}

Most of the participants could not correctly identify the exact answer of the questions on knowledge regarding indwelling urinary catheter care. Less than one-third of the subject (Mean=28.7\%) knew regarding IUC care. More than half of the participants (59\%) had poor practices regarding the frequency of catheter care per day. Most of the participants $(82 \%)$ were at the unsatisfactory level of knowledge regarding indwelling urinary catheter care (Table 4). Majority of participants $(78.7 \%)$ had a satisfactory level of practices regarding indwelling urinary catheter care (Table 5). 
Table 4. Overall level of knowledge regarding IUC care $(n=122)$

\begin{tabular}{lcc}
\hline Category & Frequency & $\%$ \\
\hline Satisfactory & 22 & 18 \\
Unsatisfactory & 100 & 82
\end{tabular}

Table 5. Overall level of practices regarding IUC care $(n=122)$

\begin{tabular}{lcc}
\hline Category & Frequency & \% \\
\hline Satisfactory & 96 & 78.7 \\
Unsatisfactory & 26 & 21.3
\end{tabular}

\section{Discussion}

A total of 122 participants took part in this study from selected wards and ICUs. The response rate was $66.6 \%$. Most of the participants $(60.7 \%)$ were less than or equal to 30 years of age. Majority of the participants $(55.7 \%)$ had three months to 5 years of work experience. Almost $82.8 \%$ of the study population had a diploma as their highest educational qualification. In Sri Lanka government nurses were recruited as a diploma nurse after the training at Nurses' Training Schools. This could be due to the busy schedule; it is not possible for nurses to find the time to do their higher studies.

\section{Knowledge and practices regarding IUC insertion}

More than one-third of the participants (36.1\%) had fair knowledge level regarding IUC insertion. One-third of the participants (32.8\%) had good knowledge level regarding IUC insertion. Almost more than three quarters $(79.5 \%)$ were in a satisfactory level of practices regarding IUC insertion and less than one quarter $(20.5 \%)$ were in an unsatisfactory level of practices. As noted in a recent Robert Wood Johnson Foundation summary, nurses' preferences and judgments are in a high position in decisions on insertion and maintenance of urinary catheters (Kurtzmann, 2007). Therefore, nurses' knowledge 
and practices regarding IUC insertion need to be at a good level.

IUC insertion is a sterile and invasive procedure (Bernard et al., 2012). A study done at Bangalore to evaluate the effectiveness of integrated catheter care pathway package regarding indwelling urinary catheter care, on knowledge of staff nurses in a selected hospital had been summarized that urinary catheters must be inserted using sterile equipment and an aseptic technique (Fernandes, 2014). In the present study, more than half of the participants (63.2\%) did not know that the catheterization is a sterile and invasive procedure. This may be the main factor for CAUTIs.

More than half of the participants $(55.7 \%)$ did not know the retained period of Foley catheter. A study done in a tertiary care centre in Thailand revealed that once inserted urinary catheter in hospitalized patients remained in place, even the indication for its use ends (Apisarnthanarak et al., 2007). Another one done at Rush University Medical Centre, to implement and evaluate the efficacy of an intervention to reduce catheter-associated urinary tract infections, revealed that once inserted, catheters tend to remain in place after appropriate indications end. That research found that overall, $32 \%$ of the catheter device days were considered inappropriate (Elpern, 2009). As per the findings of the present study, it could be argued that such incidents happened to be due to insufficient follow up of guidelines available in hospitals for health care workers. Usually, Foley catheters are used in government sector without knowing the exact retained period, which would be a problem regarding IUC caring, and also it leads to CAUTI.

Half of the participants (46.7\%) knew the correct practices and used 12-14 size urinary catheters for female patients. Less than half of the participants (45.1\%) mentioned 14-18 size urinary catheters for female patients. This would be another unnecessary problem due to the use of the inappropriate size of IUC. A study conducted by De Silva (2011) in Sri Lanka showed that using inappropriate indwelling catheter sizes cause a mechanism of injury due to pressure necrosis. In contrast to the current study findings, another study was done in Bangalore) among staff nurses in a selected hospital revealed that most of the nurses were not aware of the different sizes of catheters used for different age groups (Elpern, 2009). 
Majority of the participants correctly knew indications (mean $=5.1 \pm 0.87$ ) for catheterization. Therefore, nurses had good knowledge of indications regarding IUC insertion. These findings contrast to a study of Jane, (2015) who done a study in tertiary care hospital in India to assess knowledge and attitude among doctors and nurses regarding indication for catheterization. It revealed that the overall the knowledge of doctors was significantly better than nurses in identifying the indications for catheterization $(\mathrm{p}<0.05$, doctors mean $=8.47 \pm 0.710$, nurses mean $=6.57 \pm 0.908)$ (Jane et al., 2015). In the present study almost all the participants identified the acute and chronic urine retention (94.3\%) to monitor urine output $(86.9 \%)$ and also for presences of pressure sore and patients with incontinence $(97.5 \%)$ as indications for catheterization. A study done in India among nurses and doctors in a tertiary care hospital revealed that more than one-third of the urine output monitoring in a urine sample for a culture sensitivity was a valid indication for catheterization (Jane, et al., 2015). A study conducted in the United Kingdom revealed that majority $(89.6 \%)$ of the patients were catheterized according to the IDSA guideline indications (Cheema et al., 2012).

In the present study, most of the participants (Mean=86.5) had good practices regarding documentation after the IUC insertion. Proper documentation after the IUC insertion will help to identify when the catheter should be removed or changed. Study conduct in the United States of America revealed that the majority (87\%) of nurses documented their cases after the catheterization (Robinson et al., 2007). Another study conducted by Harley et al. (2011) showed that if there was proper documentation, it could lead to a reduction in duration (mean $=4.9 \pm 5.3$, range $1-21$ days) of the catheterization.

\section{Knowledge and practices regarding IUC care}

In the present study, the majority of the participants (82\%) had an unsatisfactory level of knowledge regarding IUC care. There were more than three quarters $(78.7 \%)$ of participants in a satisfactory level of practices regarding IUC care. A similar study conducted by Govindamma (2005) in India to assess the knowledge and practice of staff nurses regarding the care of the patient with indwelling urinary catheter showed that $42 \%$ of staff nurses had 'excellent knowledge' and $58 \%$ staff nurses had 'good knowledge' regarding indwelling 
catheter care. When compared with that study, the current study findings are higher than the results of knowledge as well as practices of urinary catheterization. A study conducted in Southeast Michigan showed that the majority of nurses (57\%) had a good level of practices regarding catheter care (Mody et al., 2010).

Concerning urinary catheter care, the study conducted among healthcare workers to assess the knowledge of recommended urinary catheter care practices in Southeast Michigan revealed that majority of respondents (85\%) were aware of recommendations, such as cleaning the catheter site regularly (Mody, et al., 2010). In contrast with those findings, the current study showed that only 50 nurses $(41 \%)$ knew the correct answer for the frequency of catheter care per day. Mody et al. (2010) showed that 35\% of health care workers know about regular changes in the catheter bag. According to the present study, less than one-third of the participants knew when a urine bag should be changed in none high-risk unit (30.3\%) and high-risk unit $(27 \%)$. Lack of knowledge on the exact time duration to change the urinary bag could lead to unnecessary infections. This result may be an indication of one of the facts for CAUTI.

\section{Conclusions}

In conclusion, CAUTI is one of the most HAIs. This is preventable if correct indications for catheterization, correct practices for catheter care and other preventive measure are followed attentively. Nurses are the most responsible parties for catheter management. Purpose of the current study was to determine the knowledge and practices of nurses regarding the insertion and care of indwelling urinary catheter in female patients. Findings of this study indicate that more than a quarter of the nurses $(31.1 \%)$ had poor knowledge regarding indwelling urinary catheter insertion. Majority of the nurses (82\%) had an unsatisfactory level of knowledge regarding indwelling urinary catheter care. Findings highlight a need for improvement of knowledge regarding urinary catheter care among nurses. This study can be improved by modifying the present study to overcome the discussed limitations. There is a need to develop proper guidelines regarding indwelling urinary catheterization and care for nurses in Sri Lanka. Regular practising of updating guideline will help to improve nurses' 
knowledge regarding indwelling urinary catheter insertion and care in Sri Lanka.

\section{Limitations}

The study was conducted in only one hospital, which was not enough to generalize the whole nurses' knowledge and practices of nurses regarding the insertion and care of indwelling urinary catheter of female patients in Sri Lanka.

\section{Acknowledgements}

The authors thank all participants for their time and commitment to this study.

\section{References}

Apisarnthanarak, A., Thongphubeth, K., Sirinvaravong, S., Kitkangvan, D., Yuekyen, C., Warachan, B., Warren, D.K., Fraser, V.J. (2007). Intervention to remove unnecessary urinary catheters in Thailand. Infection control and hospital epidemiology. http://doi:10.1086/518453

Bernard, M. S., Hunter, K. F. \& Moore, K. N., (2012). A Review of Strategies to Decrease the Duration of Indwelling Urethral Catheters and Potentially Reduce the Incidence of CatheterAssociated Urinary Tract Infections. Urologic Nursing. http: / / www.goapic.org/documents/2013/foley.pdf

Cheema, M. R., Shuaib, F. R. \& Barrett, J. A., (2012). The practice of urinary catheterization and knowledge in junior staff: a quality control study. Dove press. http://doi.org/10.2147/CA.S25554

De Silva, W. A. S. (2011). A devastating complication of the long-term indwelling catheter. Anuradhapura Medical Journal. http: / /dx.doi.org/ 10.40338/amj.v5i1.5783

Elpern, E. H., Killeen, K., Ketchem, A., Wiley, A., Patel, G., Pharm, D. \& Omar Lateef, O., (2009). Reducing the use of indwelling urinary catheters and associated urinary tract infections. American journal of critical care. http: / / doi:10.4037/ajcc2009938 
Govindamma, N. K., 2005. Assess the knowledge and practices of staff nurses regarding the care of patients with an indwelling catheter. [pdf]: Rajiv Gandhi University of Health Science. Retrieved August 05, 2016, from http://14.139.159.4:8080/jspui/bitstream/123456789/3212 / 1/Govindamma.pdf

Harley, G., Yeo, A. L., Stuart, R. L. M. F. P. \& Dendle, C. M. F. G., (2011). A Real-Life Snapshot of the Use and Abuse of Urinary Catheters on General Medical Wards. Infection Control and Hospital Epidemiology, 32, 1216-1218. http://doi:10.1086/662625

Hosseinpour, M., Noori, S., Amir-Beigi, M., Pourfakharan, M. H., Ehteram, H. \& Hamsayeh, M. (2014). Safety of latex urinary catheters for short time drainage. http://doi: 10.4103/09747796.134257

Kurtzman, E. (2007). A summary of the impact of reforms on the hospital inpatient prospective payment system (IPPS) on nursing services. [pdf] Washington: Robert Wood Johnson Foundation. Retrieved July 20, 2015, from http://www.rwjf.org/files/research/ippswhitepaper2007.pdf

Lo, E., Nicolle, L. E., Coffin, S. E., Gould, C., Maragakis, L. L., Meddings, J., Pegues, D. A., Pettis, M., Saint, S. \& Yokoe, D. S., (2014). Strategies to Prevent Catheter-Associated Urinary Tract Infections in Acute Care Hospitals: 2014 Update. Chicago Journals, [e-journal] 35(5), pp. 464-479. http://doi:10.1086/675718

Ministry of Health, (2012). Annual Health Bulletin. [pdf] Medical Statistics Unit Ministry of Health. Retrieved July 23, 2016, from http://203.94.76.60/health/eng/publications/Annual\%20He alth\%20Bulletin\%20 \%202012.pdf

Ministry of Healthcare \& Nutrition (2008). Sri Lanka Health at a glance. Retrieved May23, 2015, from http://www.statistics.gov.lk/Newsletters/Health\%20Bulletin( Medical\%20Stat).pdf

Mody, L., Saint, S., Galecki, A., Chen, S. \& Krein, S. L., (2010). 
Knowledge of Evidence-Based Urinary Catheter Care Practice Recommendations Among Healthcare Workers in Nursing Homes. Journal of the American Geriatrics Society. http://doi:10.1111/j.1532-5415.2010.02964.x

Newman, D. K. (2010). Prevention and Management of CatheterAssociated UTIs. Retrieved July 29, 2014, http://www.idse.net/download/UTI_IDSE10_WM.pdf

Parida, S. \& Mishra, S. K. (2013). Urinary tract infections in the critical care unit: A brief review. Indian Journal of Critical Care Medicine, 17(06), 370-374. http://doi:10.4103/09725229.123451

Pratt, R. \& Pellowe, C. (2010). Good practice in the management of the patient with urethral catheters. Nursing Older People, 22 (8), 25-29. http://doi:10.7748/nop2010.10.22.8.25.c7994

Robinson, A., Allen, L., Barnes, M. R., Berry, T. A., Foster, T. A., Friedrich, L. A., Holmes, J. M., Mercer, S., Plunkett, D., Vollmer, C. M. \& Weitzel, T., (2007). Development of an Evidence-Based Protocol for Reduction of Indwelling Urinary Catheter Usage. MEDSURG Nursing. Retrieved August 14, 2016, from http://lib.sjp.ac.lk/resourses/onlinej.html

Saint, S. \& Lipsky, B. A. (1999). Preventing Catheter-Related Bacteriuria. Arch Intern, Med. 159, 800-808.

Schumm, K. \& Lam, T. B. L. (2009). Types of urethral short-term voiding problems in hospitalized adults: a short version Cochrane Review. Sri Lanka Obstetrics and Gynaecology. http://doi:10.1002/14651858.CD004013.pub3

Urology Nursing Working Group, 2013. Female Urethral Catheterisation. Chatswood: Agency for Clinical Innovation. Retrieved July 1, 2014, from http://www.aci.health.nsw.gov.au/_data/assets/pdf_file/00 03/191064/ACI_Female_IDC_Jan13.pdf\#zoom=100

Wilde, M. H., McDonald, M. V., Brasch, J., McMahon, J. M., Fairbanks, E., Shan, S., Tang, W. \& Scheid, E., (2012). Longterm urinary catheter users' self-care practices and problems. Journal of Clinical Nursing, 22, pp.356-367. http://doi:10.1111/jocn.12042 
World Health Organization (WHO), (2010). The Burden of Health CareAssociated Infection Worldwide. Retrieved July 30, 2014, from http://www.who.int/gpsc/country_work/summary_2010043 O_en.pdf

Yoon, B., McIntosh, S. D., Rodriguez, L., Holley, A., Faselis, C. J. \& Liappis, A. P., (2013). Changing behaviour among Nurses to Track Indwelling Urinary Catheters in Hospitalized Patients. Interdisciplinary Perspectives on Infectious Diseases. http://doi:10.1155/2013/405041 